Meta

Journal des tradlucteurs

Translators' Journal

\title{
De vente à solde ou les dangers de l'hypercorrection
}

\section{Stanley Aléong et Christine Jourdan}

Volume 26, numéro 2, juin 1981

URI : https://id.erudit.org/iderudit/001868ar

DOI : https://doi.org/10.7202/001868ar

Aller au sommaire du numéro

Éditeur(s)

Les Presses de l'Université de Montréal

ISSN

0026-0452 (imprimé)

1492-1421 (numérique)

Découvrir la revue

Citer cet article

Aléong, S. \& Jourdan, C. (1981). De vente à solde ou les dangers de l'hypercorrection. Meta, 26(2), 148-158. https://doi.org/10.7202/001868ar d'utilisation que vous pouvez consulter en ligne.

https://apropos.erudit.org/fr/usagers/politique-dutilisation/ 


\section{PROBLÈMES ET SOLUTIONS}

\section{DE VENTE À SOLDE OU LES DANGERS DE L'HYPERCORRECTION}

Plus de deux siècles de contact intense avec l'anglais et un bilinguisme largement pratiqué ont laissé de nombreuses traces dans les vocabulaires québécois. L'anglicisme, qu'il s'agisse d'emprunts de forme, de calques ou d'emprunts de sens, a fait l'objet de nombreuses observations, voire de dénonciations. Par ailleurs, dès le milieu du XIX ${ }^{\mathrm{e}}$ siècle, le français québécois a connu également un important effort de correction lexicale tendant à substituer des syntagmes dits corrects à des expressions et termes jugés fautifs ou inappropriés. Aussi avons-nous cette longue tradition d'ouvrages guides $N e$ dites pas ... dites que l'on trouve encore en grand nombre. Si l'usage populaire n'a cure de ces efforts de correction, les traducteurs et terminologues, soucieux de l'expression juste, se doivent de rechercher la précision et l'exactitude des termes qu'ils emploient.

Dès lors que l'on se propose de corriger un item lexical, c'est-à-dire de substituer un syntagme à un autre déjà répandu dans l'usage, se pose la question de l'opportunité même du terme correctif. Encore faut-il se convaincre et convaincre les autres du caractère fautif du terme à corriger. En effet, si certains anglicismes peuvent sembler évidents, d'autres, et notamment les emprunts de sens, le sont beaucoup moins. «Comme il n'y a pas de forme nouvelle, dit Deroy (1956:215), le locuteur non averti croit aisément qu'il n'y a rien d'autre qu'une évolution sémantique régulière». Tel est le cas de plusieurs termes très répandus dans le lexique de la publicité commerciale au Québec où de vieilles traductions, aujourd'hui mises en cause, ont reçu la consécration de décennies d'usage et sont encore largement répandues dans la presse et dans les médias d'information.

Parmi les nombreux exemples de traductions malheureuses que l'on cherche à corriger, le mot vente, dans un usage particulier, est bien connu de la plupart des lecteurs. Aussi nous proposons-nous de faire, dans un premier temps, une étude de cas de ce mot et des efforts québécois entrepris afin de lui trouver un substitut acceptable; dans un deuxième temps, nous examinerons l'usage européen comparable afin de mettre en relief les différences entre les procédés québécois et européens de description d'un champ notionnel semblable. 
On sait que le champ sémantique du mot vente recouvre une grande partie de celui du terme anglais sale: action d'échanger des biens et services contre de l'argent. Or, sale en anglais comporte, entre autres, un deuxième sens, particulièrement important dans la publicité commerciale, de «vente de marchanđises à prix réduit pour une raison quelconque». Les deux sens distincts mais voisins sont différenciés tantôt par l'emploi distinctif des prépositions for et on, tantôt par le contexte. For sale veut dire simplement en vente (ex. : a house for sale), alors que on sale rend généralement la notion de prix exceptionnellement réduit (ex.: a house on sale). D'autre part, en cas d'absence de préposition, le contexte général permet de prévenir la confusion. Ainsi une phrase comme the biggest sale of the century sera décodée dans l'une ou l'autre acception suivant le sens de l'ensemble du texte.

En anglais sale, dans son acception «vente à prix réduit», s'insère dans une série paradigmatique très riche, d'autant plus que l'ordre syntaxique déterminant/déterminé autorise de nombreuses combinaisons. Ainsi avons-nous trouvé dans les pages des quotidiens montréalais de langue anglaise les expressions suivantes que l'on peut classer grosso modo dans quatre catégories :

1. Les motifs de la vente exceptionnelle

\begin{tabular}{l|l} 
opening & \\
closing & \\
going-out-of-business & \\
bankruptcy & \\
clearance & \\
liquidation & \\
fire & \\
water & sale \\
smoke & \\
super-saver & \\
anniversary & \\
red tag & \\
inventory & \\
bargain & \\
one cent & \\
one dollar & \\
rummage & \\
renovation & \\
manager's & \\
surprise & \\
discount & \\
half price & \\
sacrifice, etc.
\end{tabular}

2. Le lieu de la vente exceptionnelle:

$$
\begin{aligned}
& \text { storewide } \\
& \text { trans-Canada } \\
& \text { warehouse } \\
& \text { sidewalk } \\
& \text { garage } \\
& \text { apartment, etc. }
\end{aligned}
$$


3. Le moment, vu comme motif de la vente exceptionnelle :

annual
Christmas
Boxing day
Winter
Spring
Summer
Fall
end-of-season
back-to-school
breakfast, etc.

4. L'objet même de la vente peut être annoncé aussi :

car
stereo
speaker
book
house
watch
end-of-line
silver-plating
shoe
furniture
suit, etc.

Signalons enfin que nombre de commerçants apposent à leur vitrine tout simplement sale, sans autres qualificatifs, voulant dire ainsi que leurs prix sont réduits. Certains commerces arborent cette affiche en permanence, comme si leurs prix étaient toujours réduits.

Dès la fin du XIX $\mathrm{XI}^{\mathrm{e}}$ siècle, au mot vente en français québécois va se joindre cet usage pris à l'anglais. Que l'on appelle ce phénomène calque sémantique ou emprunt de sens, suivant la terminologie que l'on préfère, il s'agit d'une forme d'extension du champ sémantique du mot. Dans les journaux montréalais de la fin du siècle dernier, on trouve de nombreux exemples de l'emploi du mot vente, tantôt dans son acception générale, tantôt dans son acception particulière dont il est ici question. À la page 2 de La Presse du 20 février 1886, par exemple, nous observons que la Compagnie de prêts et de crédit foncier annonce une grande vente par encan, tandis que le 24 février suivant, à la page 3 du même journal, D. McCormack, bottines et souliers, annonce une grande vente à bon marché. Le $1^{\text {er }}$ février 1887, à la page 3 de La Presse, la Compagnie de prêt et de crédit foncier annonce une vente extraordinaire. Toujours à la même date mais en page 4, la maison Alphonse Valiquette signale une grande vente annuelle à une réduction spéciale de $50 \%$. Le 12 février 1887 , en page 6 du même journal, nous trouvons une de nos ventes les plus importantes chez Boisseau frères, tissus et vêtements. En page 3 du 26 février 1887, il y a une grande vente sans réserve chez Alphonse Valiquette. Dans l'année 1893, La Presse contient de nombreux exemples de vente: grande mise en vente (4 février, p. 2); grande vente à bon marché ( 6 février, p. 3 ); la vente spéciale de chaussures (10 février, p. 2); vente à sacrifice (11 février, p. 3); grande vente de liquidation (16 février, p. 3 ) ; ...notre grande vente à escompte (17 février, p. 6). 
Nous pourrions multiplier les exemples. Une observation s'impose : parallèlement à l'usage général du mot vente, il se développe peu avant la fin du siècle dernier et au contact de l'anglais, un autre sens de vente en français québécois. On pourrait arguer certes que certains des exemples cités plus haut n'ont rien de particulier; c'est vrai, mais il est également évident que pour certains utilisateurs, vente avait d'ores et déjà acquis le sens de «vente de marchandises à prix réduit ».

Dès lors que vente avait acquis cette signification empruntée à l'anglais, les séries paradigmatiques comparables à celles du sale anglais lui étaient ouvertes. Des formules aussi saugrenues que vente de fumée, vente d'eau, allaient devenir tout à fait intelligibles. En nous rapportant à la liste ci-dessus d'exemples de types de sale, il suffirait de traduire les expressions anglaises à l'aide de vente pour retrouver bon nombre d'usages encore très actuels dans le français québécois. On a vu une compagnie aérienne vanter sa super vente de tarifs et certains magasins faire des ventes à un cent. Le grand quotidien La Presse a actuellement dans ses petites annonces une rubrique vente de garages. Il ne s'agit pas, on s'en doute bien, de garages à vendre, mais plutôt de ventes d'objets d'occasion chez des particuliers. Ainsi, il nous a été donné de photographier, rue Beaubien à Montréal, l'enseigne d'un magasin affichant allègrement: «pas de vente, juste des bas prix».

Cette évolution sémantique particulière du mot vente en français québécois n'a pas échappé à la surveillance des puristes et autres défenseurs du bon langage. À cet égard, il est curieux de constater que le célèbre abbé Étienne Blanchard, pourtant grand pourfendeur de l'anglicisme au Québec, consacre peu de lignes à la question. Il traduit bargain par solde (Blanchard, 1919:16) et bargain day par soldes (Ibid. : 76). Par ailleurs il propose vente d'occasion pour bargain sale (Blanchard, 1928: 281). Plus près de nous, Augustin Turenne trouve erronées les expressions vente à prix réduits et vente de clairance et préfère proposer comme équivalents vente au rabais et (sic) soldes. Gérard Dagenais consacrera une page à ce problème (1968: 1196), alors que Gilles Colpron (1970: 86) en parlera en deux lignes. Victor Barbeau dénonce en 1970 les anglicismes vente de feu, vente de fumée et vente d'eau (Barbeau, 1970: 155).

Le raisonnement de ces critiques est à peu près le même : le mot vente en bon français n'a pas le sens anglais de vente à prix réduit. Suivant ce point de vue, une expression usuelle comme prix de vente ne peut pas comporter le sens de prix exceptionnellement bas (c'est-à-dire par opposition au prix courant). De même, comme il va de soi que le but du commerce est de vendre, le mot vente affiché dans la vitrine est absolument inutile. Il s'agirait plutôt d'une mauvaise traduction de l'anglais. Quant à des formules comme vente de feu, vente de Noël ou la célèbre vente d'écoulement de blanc à la verge, il va sans dire qu'elles sont toutes condamnées comme étant illogiques et contre les principes de la langue. Selon ce point de vue, une phrase très courante comme c'était pas cher, je l'ai eu en vente serait redondante et erronée.

Mais c'est une chose que de dénoncer les ravages de l'anglicisme de sens; c'est autre chose que de proposer des solutions de rechange. Phénomène clas- 
sique que connaissent tous les traducteurs, le sale anglais possède une grande souplesse d'usage, ou, plus précisément, possède de nombreux contextes d'emploi, qui rendent la traduction malaisée. De même, vente se prête à des combinaisons aussi différentes que vente des tarifs Air Canada, vente d'ouverture et vente d'entrepôt.

Une façon de procéder était de qualifier le mot vente à l'aide d'un adjectif ou d'un complément. Car en fait, il s'agit d'une sorte de vente, mais dans des conditions particulières. Des expressions comme vente à rabais, vente exceptionnelle, vente-réclame ou vente extraordinaire sauraient bien rendre la notion de vente à prix réduit.

L'autre façon de procéder était de faire appel à des termes ou expressions qui seraient plus justes ou adéquats. Parmi ces termes dont il sera question plus bas, celui qui connaîtra le plus grand succès sera solde et son pluriel soldes. S'il est difficile de situer chronologiquement l'avènement de solde dans le vocabulaire québécois, nous avons vu que dès 1916 l'abbé Blanchard traduisait bargain par solde et bargain days par soldes. Pour Victor Barbeau le terme juste était solde ou occasion (Barbeau 1970 : 155).

Dans son célèbre ouvrage, Gérard Dagenais nous dit en parlant de vente: «Ce qu'on veut dire, c'est vente à rabais ou solde» (Dagenais, 1968:631). Plus loin, en parlant de solde, l'auteur nous dit: "C'est aussi l'action d'offrir une quantité de marchandise à un prix inférieur au prix ordinaire» (Ibid:632). Pour cet auteur donc, le mot solde est l'équivalent de vente au sens critiqué. Chez un autre auteur, notre grande vente d'été doit se traduire par vente au rabais, vente de soldes, solde (Colpron, $1970: 86$ ).

Dans une brochure intitulée Le vocabulaire de la vente promotionnelle, et publiée par l'Office de la langue française (1974), plusieurs pages sont consacrées à la traduction des expressions anglaises avec sale. Pour bargain sale, l'Office propose: vente au rabais, vente à rabais, (vente en) solde, vente de soldes (p. 9). Clearance doit se traduire par (vente de) liquidation, (vente en) solde et vente de soldes (p. 9). À la même page, les auteurs nous disent : «Alors que la liquidation consiste toujours en une vente de soldes, un solde peut ne pas être spécifiquement «en vue d'un écoulement rapide de marchandises», mais consister en une simple vente au rabais» (p. 10). Plus loin, closing down sale doit se traduire par solde de fermeture ou solde de clôture (p. 12). Inventory clearance est traduit par solde d'inventaire, liquidation de stock après inventaire, ou solde après inventaire (p. 16). Quant à la traduction de sale tout court, on trouve vente au rabais, vente à rabais, (vente en) solde, vente de soldes, vente-réclame, vente de liquidation (p. 17). Renovation sale a comme pendant solde de rénovation ou vente-réclame de rénovation ( $\mathrm{p} .16)$. On notera enfin que storewide sale se traduit par solde général (p. 20) et warehouse sale par solde à l'entrepôt (p. 21).

D'après ces traductions, il est évident que pour les auteurs de cette brochure, terminologues de l'Office de la langue française, solde est l'équivalent de vente au sens de vente à prix réduit. Ne disent-ils pas explicitement : «Note : un 
solde sera tantôt une simple vente au rabais, tantôt une vente de liquidation. Dans la terminologie de la vente promotionnelle «solde» est toujours masculin » (p. 17).

Ces prises de position à caractère officiel en faveur du mot solde confirment une évolution déjà amorcée vers le début du siècle et qui a donné au mot solde en français québécois le sens général de vente à prix réduit. Fort de l'appui tant des puristes que d'un organisme officiel, solde semble voué à remplacer un jour vente. Des exemples récents semblent démontrer que tel est le cas chez nombre d'utilisateurs. Dans un entrefilet du 12 décembre 1979 de La Presse (p. A-19), un journaliste écrit : «Le préposé aux réservations d'Air Canada responsable de la diffusion du système préférentiel du solde de places préconisé par Air Canada a été suspendu préalablement au renvoi...» Plus loin dans le rnême article : «... est d'autant plus déçu de la décision d'Air Canada qu'il se dit très favorable à la décision d'organiser un solde pour améliorer le coefficient cle remplissage à bord des avions en période creuse».

À partir du moment où solde paraît le synonyme de vente, toutes les formules construites avec vente peuvent être combinées avec solde. On ne s'étonne guère de lire actuellement dans la presse montréalaise des formules comme solde $30^{e}$ anniversaire, solde de meubles, solde petit déjêuner, ou grand solde de téléviseurs au Centre d'aubaines Eaton à l'entrepôt. Aussi voit-on dans les magasins de plus en plus d'étiquettes en solde ou à solder.

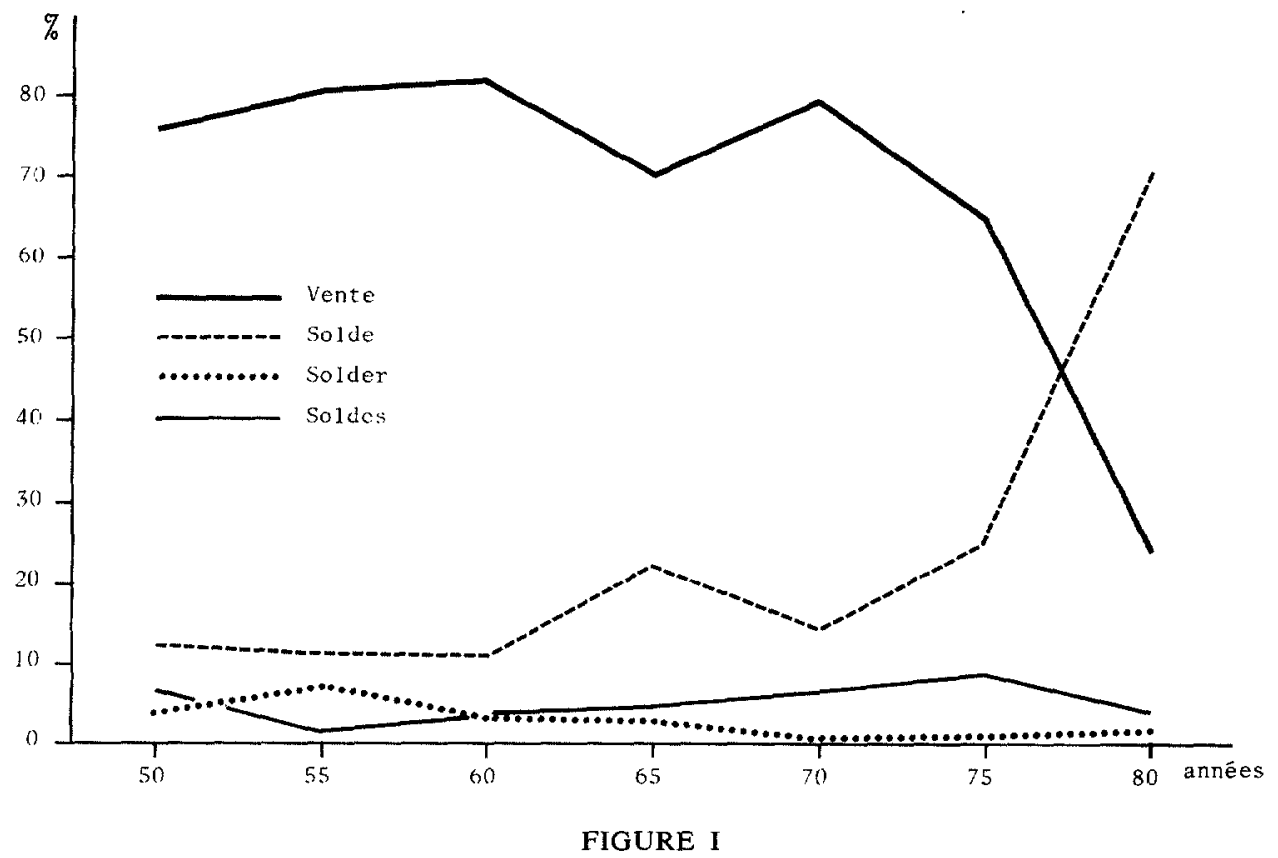

Pourcentages relatifs des termes vente, solde, solder et soldes dans les quinze premiers jours de janvier de 1950 à 1980 dans La Presse. 
Afin de savoir si solde était vraiment en passe de remplacer vente dans l'usage, nous avons fait un relevé statistique des usages des 15 premiers jours de janvier dans La Presse, depuis 1945 jusqu'en 1980, à raison d'intervalles de 5 ans. Nous avons contrôlé, d'une part, toutes les formes, simples et composées, de vente, au sens qui nous intéresse, et, d'autre part, toutes les occurrences de solde, solder et soldes. Nous avons trouvé par exemple, le 2 janvier 1950 en page 2, une vente annuelle de mi-hiver de fourrures chez Morgan, cependant qu'à la page 16 on observe Souliers à solder, Dupuis solde paletots d'hiver, coupe vents.

Comme on le voit sur le graphique, le terme vente est de loin le plus utilisé jusqu'au début des années 70. Ensuite commence une période de déclin de la fréquence relative de vente, cependant que, parallèlement, le terme solde, son concurrent direct, connaît une forte progression. Si cette tendance se maintient, il ne semble pas impossible que dans un avenir assez proche solde ne remplace définitivement vente.

Reste à savoir si le terme solde est le plus juste comme correctif de vente. Certes il est vrai qu'à partir du moment où la majorité des utilisateurs de la langue considèrent que solde est une «bonne» correction de vente, la question même de la justesse de la correction devient presque caduque. Par ailleurs, il est également vrai que bon nombre de grammairiens québécois ainsi que l'Office de la langue française voient dans l'usage européen le modèle du bon français québécois. Il sera donc intéressant d'examiner comment le français d'Europe décrit des réalités semblables.

On constate dès le départ que chez les Français le mot vente tout seul n'a jamais, ou du moins pas encore, acquis le sens de vente à prix réduit. La publicité commerciale française fera plutôt appel à une gamme de mots parmi lesquels nous trouverons des formules élaborées à partir de vente et de mots différents.

Puisque les grammairiens québécois ont trouvé dans solde une solution au problème de la correction de vente, il est intéressant d'examiner l'usage européen de ce terme. Cependant, pour comprendre les usages actuels, il est important de faire quelques brefs rappels historiques. Dès le milieu du XIX ${ }^{\mathrm{e}}$ siècle, les dictionnaires français relèvent deux sens apparentés mais distincts de solde. Il s'agit d'une part d'un terme de commerce désignant la différence entre le débit et le crédit d'un compte. Les comptables parlent alors de solde débiteur, solde créditeur, solde d'un compte, solde de facture, etc. C'est ce sens de solde que relèvent par exemple A. Clas et P. Horguelin (1970: 23). D'autre part, toujours dans la deuxième moitié du XIX ${ }^{e}$ siècle, solde en est venu à désigner, selon le Littré, «marchandises qui restent en magasin à la fin d'une saison et qui, démodées ou défraîchies, se vendent au rabais» (Littré, 1971: t. 7, p. 231). Vendre en solde veut dire «vendre avec diminution de prix des marchandises restées en magasin ».

L'accent doit être mis ici sur le sens de «marchandise restant en surplus et devant être vendue au rabais». Le mot solde désigne en fait la marchandise et nullement l'action. Le verbe doit être compris donc comme l'action de vendre 
à prix réduit des marchandises restant à la fin de la saison. Ainsi les Grands magasins Au Bon Marché annoncent dans leur agenda-buvard de 1896, à la page 9 :

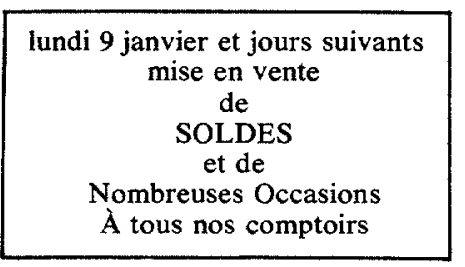

C'est ce même sens, sans exception, que nous retrouvons dans tous les dictionnaires récents consultés. Par exemple le Grand Robert de 1966 reproduit la définition de solde qu'a donnée le Littré avec, comme exemples marchandise, articles vendus en solde, mis en solde. L'auteur ajoute la remarque suivante: «L'expression s'emploie surtout en parlant de tissus, vêtements, chaussures, etc.» (Paul Robert, 1966: t. 6, p. 282). Dans son Encyclopédie du bon français dans l'usage contemporain, Dupré écrit à propos de solde: «se dit aussi de marchandises qui, restant invendues, sont liquidées au rabais par le fabricant ou le marchand. Vendre en solde» (Dupré, 1972: 2405). Au sujet du genre grammatical de solde, source de confusion, Adolphe V. Thomas, dans son répertoire de difficultés de la langue, écrit : «solde est masculin en terme de commerce..., et particulièrement au sens de "marchandise vendue au rabais": Acheter un solde intéressant » (Thomas, 1971: 387). Dans la dernière édition du Petit Robert (1977), la définition est presque identique : "marchandises qui se vendent au rabais. Articles vendus en solde, mis en solde» (Paul Robert, 1977; 1828). Pour le Harraps, solde se traduit ainsi : «(b) (Vente de) soldes (1) clearance sale; (ii) bargain counter. Soldes après inventaire, stock-taking sale. S. d'édition (i) remainder(s), remainder line (in books). '(En) solde' 'to clear' Prix de s., bargain prices" (Mansion, J. E., 1970: Part 1, p. 790).

Suivant le même dictionnaire, sale se traduit par: «2. Com. : (Bargain)sale, vente de réclame, au rabais; liquidation f. Closing-down sale, soldes des $(\mathrm{m})$ avant départ; liquidation du stock avant départ. The sales are on, c'est le moment des soldes. To buy goods at the sales, acheter des soldes. Sale goods, (i) soldes; (ii) marchandises f. de pacotille. Sale price, prix m. de solde» (Ibid.: JPart 2 : 1079).

Force est de constater que dans les dictionnaires unilingues ou bilingues, on ne fait jamais mention de solde au sens de «action de vendre à prix réduit». Tout au plus doit-on parler d'une convergence de forme dans une formule comme prix de solde. De toute évidence, chez les Européens solde désigne exclusivement la marchandise vendue à prix réduit. Mais afin de vérifier si l'usage réel correspondait à la définition du dictionnaire, nous avons contrôlé également la publicité commerciale du journal parisien Le Monde au cours des quinze premiers jours de janvier, depuis 1950 jusqu'en 1980, par intervalle de 5 ans. Nous avons relevé tous les emplois de vente, de solde, de solder et de soldes. À propos de l'usage français de vente, nous rappellerons que nous n'avons trouvé aucun exemple de l'usage particulier dont il est question en français québécois. 


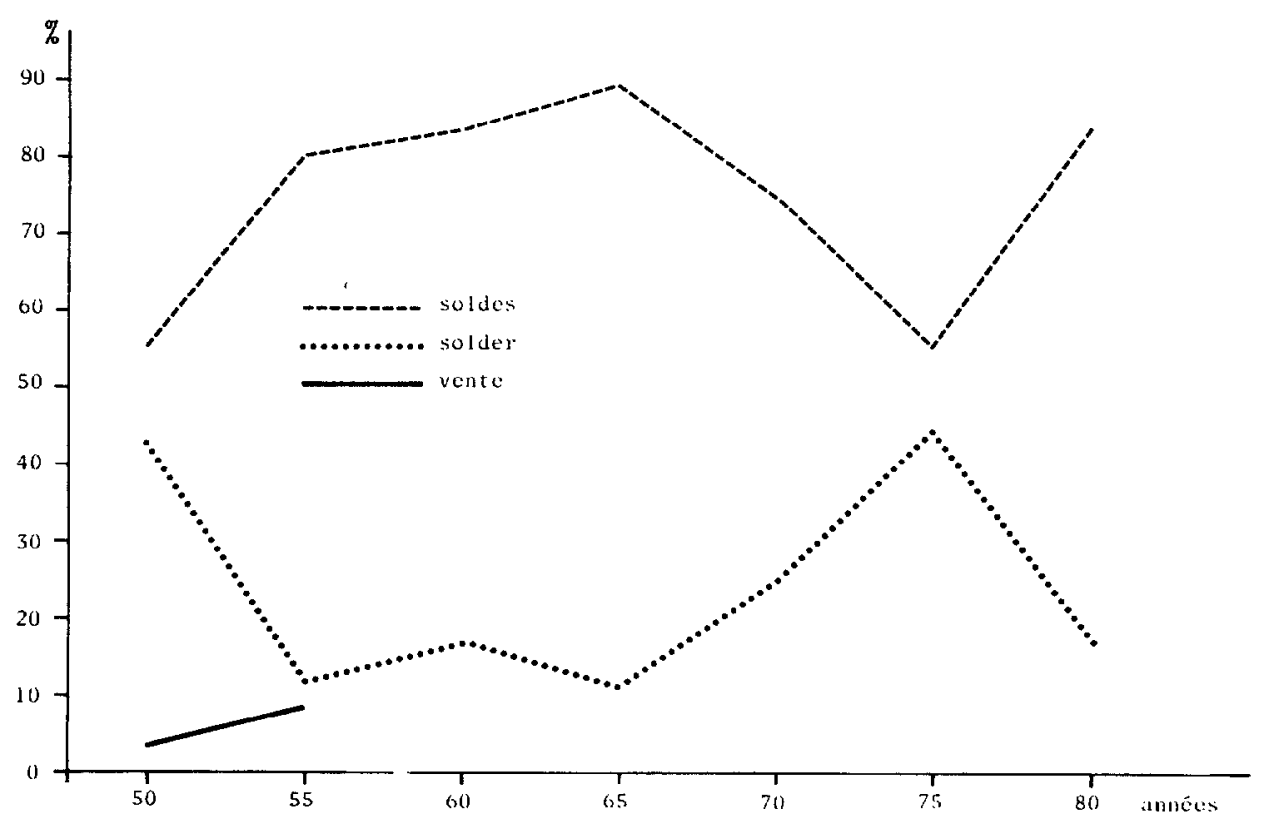

FIGURE 2

Pourcentages relatifs des termes soldes, solder et vente dans les quinze premiers jours de janvier de 1950 à 1980 dans Le Monde.

D'après la figure 2, nous voyons que le terme le plus répandu dans l'usage français est soldes, suivi du verbe solder et de vente. Par exemple, à la page 12 du Monde du 4 janvier 1950, on trouve: Fourrures Maurice, soldes annuels, après inventaire. Le 2 janvier 1950 à la page 5, Henri Halphen solde à ses rayons de dames, fillettes, jeunes gens, et dans la page 12 du 6 janvier 1950: Old England a reçu des tweeds. Vente spéciale de janvier. Prix modérés.

Les exemples 1980 vont tous dans le même sens. Soldes, au sens de marchandises à prix réduit, est de loin le terme le plus répandu, suivi du verbe solder. Fait significatif, nous n'avons pas trouvé un seul exemple de solde au sens de "vente à prix réduit». Par contre, nous avons trouvé beaucoup de vente, qualifiée certes de spéciale, exceptionnelle, extraordinaire, amiable ou de vente-réclame et de exposition-vente.

Force est donc de constater que l'usage québécois de solde, correctif de vente, semble être un pur néologisme qui a peu à voir avec l'usage européen. Tout se passe comme si les correcteurs québécois, y compris l'Office de la langue française, avaient tout simplement transféré vers solde le sens critiqué de vente, sans se préoccuper outre mesure de l'acception originelle de solde. Sans vouloir parler d'erreur, puisque pour bon nombre d'usagers québécois solde est vraiment perçu comme un correctif de vente, nous dirions que, si vente est un anglicisme, solde l'est aussi. Ne s'agit-il pas d'une forme d'hypercorrection? 
Il reste à voir comment l'usage européen découpe et lexicalise le champ notionnel que recouvrent les usages québécois de vente et de solde. Là où l'anglais et le français québécois disposent d'un terme passe-partout qui peut s'appliquer aussi bien à des fromages, des vêtements, des places d'avion qu'à des livres et des alcools, le français européen fait appel à des termes différents suivant la nature du produit et des nuances de sens à rendre. Sans avoir la prétention de vouloir faire le tour de tous les procédés du langage publicitaire français, nous avons relevé au cours de notre enquête les mots clefs suivants dont certains sont pratiquement inconnus au Québec :

1. Soldes et solder. Ces termes n'ont plus guère besoin de définition. Rappelons toutefois qu'il s'agit presque toujours de marchandises vendues en fin de saison ou en vue de renouveler les stocks.

2. En réclame, vente-réclame. Vente spéciale mettant en vedette un produit particulier. Par exemple : En réclame cette semaine, le pantalon Unisex; Articles en réclame.

3. En promotion, promo, vente promotionnelle, tarif ou prix promotionnel. Ces termes sont parmi les plus répandus et correspondent à une grande partie de l'usage québécois de vente. Ils s'appliquent à toute vente à prix réduit à caractère promotionnel, qu'il s'agisse de beurre, de fourrures ou de voitures. Par exemple : notre promotion du mois, notre rayon promotions, nos promotions de Noël, tarif promotionnel Paris-Montréal. Il est intéressant de noter que l'Office de la langue française a intitulé sa brochure Le vocabulaire de la vente promotionnelle alors qu'à l'intérieur, ce dernier terme n'est jamais employé.

4. Baisse, grande baisse, baisse exceptionnelle. Par exemple : grande baisse sur le cuir; baisse exceptionnelle sur les livres.

5. Prix démarqués, grandes démarques. Il s'agit de termes qui recouvrent approximativement l'expression québécoise prix coupés. Ils s'appliquent particulièrement aux vêtements et aux objets usuels, mais leur champ d'extension est très grand.

6. Remises exceptionnelles, grandes remises. Par exemple : remises exceptionnelles pendant la durée de l'exposition.

7. La quinzaine de..., la semaine de..., le mois de... On indique ainsi l'existence de prix spéciaux pendant la période indiquée. Par exemple: la quinzaine du cuir, la semaine de la cuisine.

8. Braderie, brader. L'action de liquider à très bas prix. Ces termes peuvent remplacer avantageusement vente ou solde dans bien des contextes. Par exemple : pendant trois jours, grande braderie.

9. Prix dégriffés,... dégriffés. Il s'agit de vêtements de grandes marques dont on a enlevé le label, permettant ainsi au marchand de les vendre à des prix réduits. Par exemple : costumes et foulards dégriffés.

10. Occasions, occasions exceptionnelles. Par exemple : cette semaine, grandes occasions dans l'électroménager; occasions exceptionnelles dans le bois.

11. Fins de séries. Il s'agit de marchandises dont la fabrication a cessé ou qui ne seront plus gardées en stock. De ce fait, elles sont vendues à bas prix. Par 
exemple: profitez de nos fins de séries. Note: Au Québec les fins de séries sont souvent appelées des «lignes discontinuées".

12. Réalisation de stock: L'équivalent de liquidation. Par exemple : Grande réalisation de tout notre rayon hommes.

S'ajoutent à ces formules stéréotypées des termes qui, en France comme au Québec, appartiennent au vocabulaire général de la réclame: prix chocs, prix massacrés, offres spéciales, liquidation, etc.

L'histoire de l'usage québécois de vente et de solde illustre combien il est difficile d'éviter le calque sémantique dès que le traducteur raisonne au niveau des seuls mots. Certes pour nombre de locuteurs d'aujourd'hui, solde est une nette amélioration par rapport à vente, phénomène que l'on ne doit pas méconnaître. Il est également vrai que, au-delà de la question de la perception de l'opportunité d'un mot par rapport à un autre, nous sommes devant un phénomène plus vaste, celui de la différence entre les traditions et les pratiques commerciales de la France et du Québec. On ne saurait alors assez répéter que la bonne traduction est plus que la simple mise en parallèle de deux séries de termes; tout au contraire, «l'équivalence des textes repose sur l'équivalence de situations» (Vinay, Darbelnet, $1967: 22$ ).

\section{Stanley Aléong Christine Jourdan}

\section{BIBLIOGRAPHIE}

BARBEAU, V., le Français au Canada, Montréal, Garneau, 1970.

BLANCHARD, E. (Abbé), le Bon Français en affaires, Montréal, Étienne Blanchard, 1919. - Bon langage, Montréal, Librairie Beauchemin Ltée, 1928.

CLAS, A. et P. HORGUELIN, le Français, langue des affaires. Textes et vocabulaire, Montréal, McGraw-Hill, 1970.

COLPRON, G., les Anglicismes au Québec, Montréal, Librairie Beauchemin Ltée, 1970.

DEROY, L., l'Emprunt linguistique, Paris, Société d'éđition «Les Belles Lettres», 1956.

DUPRÉ, Encyclopédie du bon français dans l'usage contemporain, Paris, Éditions de Trévisse, 1972.

LITTRÉ, E., Dictionnaire de la langue française, Paris, Gallimard/Hachette, 1971.

MANSION, J. E. (rédacteur), Harrap's Standard French and English Dictionary, London, George G. Harrap \& Co. Ltd., Part 1 : Fr.-Eng. ; Part 2: Eng.-Fr., 1970.

OFFICE DE LA LANGUE FRANÇAISE, Vocabulaire général de la vente en magasin. La vente promotionnelle, Québec, Éditeur officiel, 1974.

- Petit Robert ou Dictionnaire alphabétique et analogique de la langue française, Paris, Société du Nouveau Littré, 1977.

THOMAS, A. V., Dictionnaire des difficultés de la langue française, Paris, Larousse, 1956.

TURENNE, A., Petit dictionnaire du «joual » au français, Montréal, Éditions de l'Homme, 1962.

VINAY, J.-P. et J. DARBELNET, Stylistique comparée du français et de l'anglais, Montréal, Librairie Beauchemin Ltée, 1967. 